\title{
Breakdown mechanism of metal halide lamps
}

\author{
Hiroshi WASHIMI*
}

\begin{abstract}
This paper describes breakdown mechanism of the metal halide lamp containing Sodium, Scandium and Thorium iodides in DC operation. Current growth, voltage collapse and the formative time lag of a discharge were measured. The time lag was compared with the calculated drift time of both electrons and ions in order to presume the dominant secondary process. It was found that a Townsend-type breakdown with $\gamma_{i}$ process occurs in the starting gap and that two types of breakdown, i.e. (1) prebreakdown $\rightarrow$ arc and (2) prebreakdown $\rightarrow$ glow $\rightarrow$ arc occur in the main gap. Which type will occur depends on the burning period, the impressed voltage and its polarity. Townsend mechanism with $\gamma_{i}$ process and/or $\gamma_{p}$ process as the dominant secondary process has an important role for the breakdown. On the comparison with the results in case of the mercury lamp, the appearance of the prebreakdown stage and the glow-to-arc step discharge are principally considered to be due to the suppression of electron multiplication byl the electron attachment and/or quenching in impurity gases.
\end{abstract}

\section{Introduction}

Metal halide lamps ${ }^{13}$ seem to be the most promising lamps because of high efficacy, high color rendition and a variety of lamp types with different physical and optical characteristics for various applications. However, they have some problems to be solved. One of the problems is, for example, their relatively high starting voltage which requires use of specific starting equipment.

Therefore, the development of new metal halide lamp which is compatible with ordinary mercury lamp ballasts is regarded as of major importance nowadays because of energy saving. For the development of such lamp, more detailed research on lamp starting mechanism is necessary.

As is generally known, starting process of metal halide lamps consists of breakdown in the starting gap followed by breakdown and glow-to-arc transition in the main gap ${ }^{1)}$. There exists a close relation between breakdown voltage in the starting gap and glow-to-arc transition voltage in the main gap $^{23}$. Breakdown in metal halide lamps is a statistical phenomenon, then theoretical and experimental studies on the statistical distribution of the breakdown voltage have been made ${ }^{3}$.

The purpose of the study described in this paper is to find the breakdown mechanism in the metal halide lamp. Current growth through a gap, voltage collapse across a gap and the formative time lag of

* Engineering Section, Lamp Department, Minakuchi Plant, NEC Sylvania Corporation, 3-1, Nichiden, Minakuchi-cho, Koka-gun, Shiga a discharge were measured by impression of DC impulse voltage to arc tubes of metal halide and mercury lamps and then the time lag was compared with electronic and ionic drift times in order to presume the dominant secondary process. From these results we discussed the breakdown mechanism of the metal halide lamp.

\section{Experimental Procedure}

In this paper, a gap between main electrodes ( $A$ and $B$ in Fig. 1) is defined as the main gap and an auxiliary gap between starting electrode (probe C in Fig. 1) and adjacent main electrode (A) as the starting gap.

Fig. 1 and Fig. 2 show electrical circuits for measuring current growth, voltage collapse and the formative time lag in the gaps of the metal halide lamp.

Oscillograms of the current growth and the voltage collapse were measured by impression of DC impulse voltage to the starting gap after impression of a constant DC voltage to the main gap.

The formative time lag was measured for the lamp burned for several hours and was experimentally determined by the time difference between probe and main currents displayed on an oscilloscope.

Pure argon of 35 Torr, mercury, and metal halide additives $\left(\mathrm{ScI}_{3}, \mathrm{NaI}\right.$ and $\left.\mathrm{ThI}_{4}\right)$ are filled in the arc tube of 400 Watt metal halide lamps used in this experiment. These lamps were aged about one hour before measurements. Experiments were carried out at room temperature. 

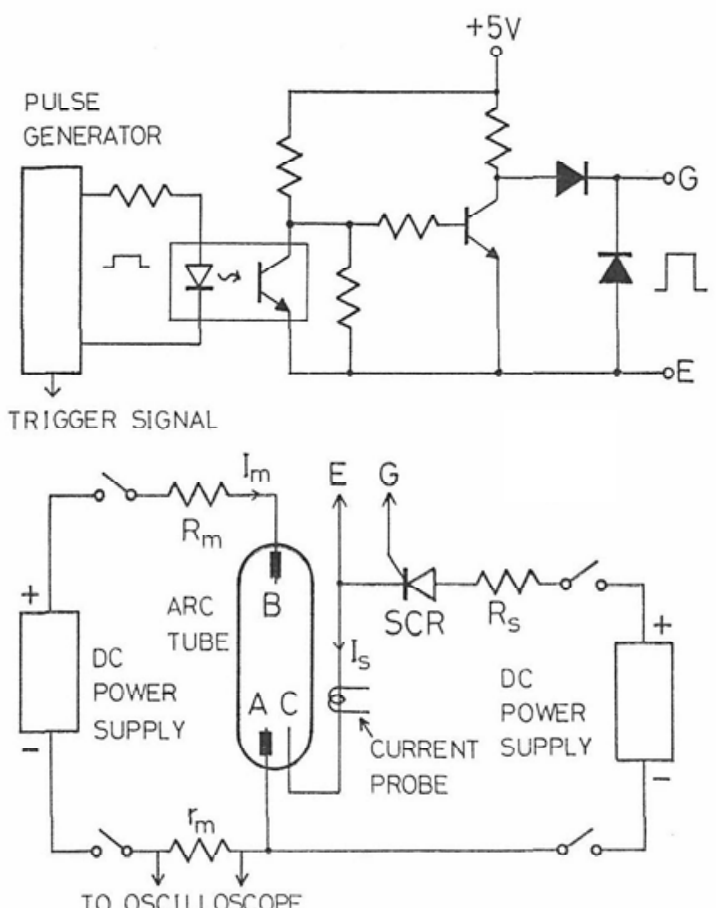

Fig. 1 Electrical circuits for measuring the formative time lag in the main gap where the main electrode $A$ operates as a cathode.

$I_{s}$ : probe current, $I_{m}$ : main current

TO OSCILLOSCOPE

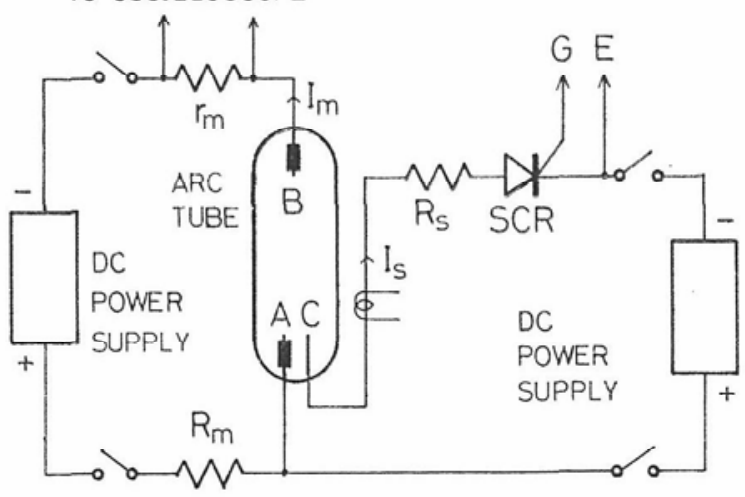

Fig. 2 Electrical circuit for measuring the formative time lag in the main gap where A operates as an anode.

\section{Results}

\subsection{Current and voltage waveforms in electrical breakdown process}

(a) In case of the main electrode A as a cathode

Fig. 3 shows a typical current growth in the main gap where the adjacent main electrode $\mathrm{A}$ operates as a cathode.

At low impressed voltage $V_{A B}$, a first stage discharge with small current of about $100 \mu \mathrm{A}$ appeared as illustrated in Fig. 3(a) and a weakly luminous glow appeared indistinktly all over the gap. The first stage discharge continued only for the duration of the auxiliary discharge in the starting gap. Further multiplication of the current followed by breakdown of the main gap did not occur. It seems that accumulation of the space charge is not great enough to promote the breakdown in this stage. The first stage discharge is considered to be a kind of prebreakdown phenomenon.

With increasing $V_{A B}$, the discharge current increased suddenly after some delay (Fig. 3(b)) and the first stage discharge transited to breakdown of the gap. Discharge phenomenon after the breakdown was either a glow discharge (the second stage) or an arc discharge (the final stage) as will be described later. Fig. 3(b) is an example followed by the glow discharge which is stable in such circuit condition as high series resistance of $R_{m}(=10 \mathrm{~K} \Omega)$. If $R_{m}$ is small value and the electric power density supplied to the cathode is large enough to form an arc spot, glow-to-arc transition occurs easily ${ }^{4}$.

At higher overvoltage, the duration of the first stage discharge extremely decreased and the first stage discharge transited rapidly to the second stage discharge as shown in Fig. 3(c).

We observed the following two types of breakdown in the metal halide lamp: those are, (1) prebreakdown $\rightarrow$ arc and (2) prebreakdown $\rightarrow$ glow $\rightarrow$ arc. The type (1) was apt to occur in the lamp burned

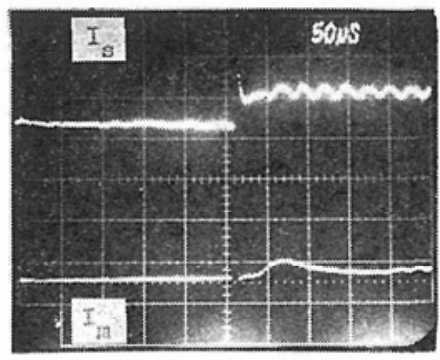

(a)

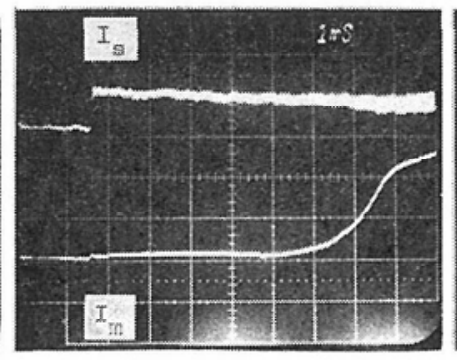

(b)

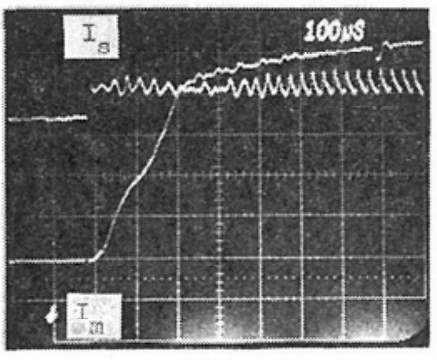

(c)

$V_{A C}=380 \mathrm{~V}$

$V_{A C}=380 \mathrm{~V}$

$V_{A C}=380 \mathrm{~V}$

$V_{A B}=400 \mathrm{~V}$ (overvoltage: $\sim 7 \%$ )

$V_{A B}=450 \mathrm{~V}(\sim 20 \%)$

$I_{m}: 5 \mathrm{~mA} /$ div.

$V_{A B}=500 \mathrm{~V}(\sim 33 \%)$

$I_{m}: 5 \mathrm{~mA} /$ div.

Time scale: $1 \mathrm{~ms} /$ div.

Time scale: $100 \mu \mathrm{s} /$ div. Time scale: $50 \mu \mathrm{s} /$ div.

Fig. 3 Typical current growth in the main gap where $A$ operates as a cathode. $I_{s}$ (probe current) $5 \mathrm{~mA} /$ div. 


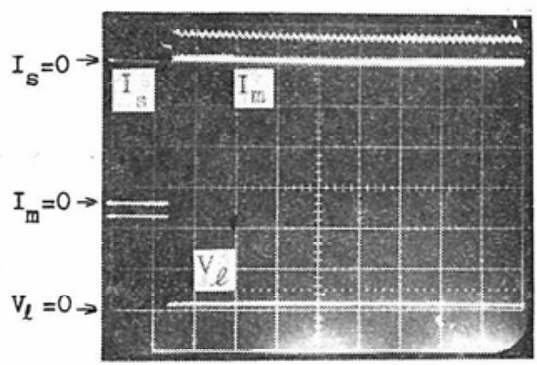

(a)

$I_{s}: 10 \mathrm{~mA} / \mathrm{div}$.

$I_{m}: 1 \mathrm{~A} /$ div

$V_{l}: 200 \mathrm{~V} /$ div.

$V_{A G}=400 \mathrm{~V}$

$V_{A B}=450 \mathrm{~V}$

Time scale: $100 \mathrm{~ms} / \mathrm{div}$.

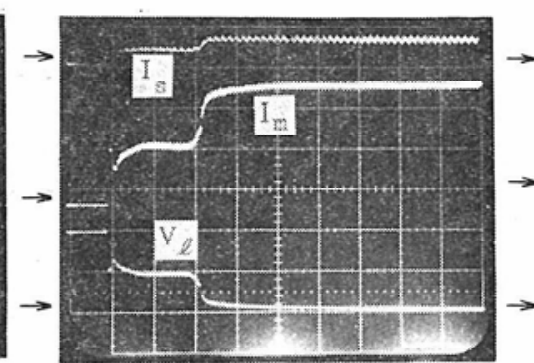

(b)

$I_{s}: 10 \mathrm{~mA} /$ div.

$I_{\mathrm{m}}: 1 \mathrm{~A} / \mathrm{div}$.

$V_{l}: 200 \mathrm{~V} /$ div.

$V_{A C}=400 \mathrm{~V}$

$V_{A B}=400 \mathrm{~V}$

Time scale: $100 \mathrm{~ms} /$ div.

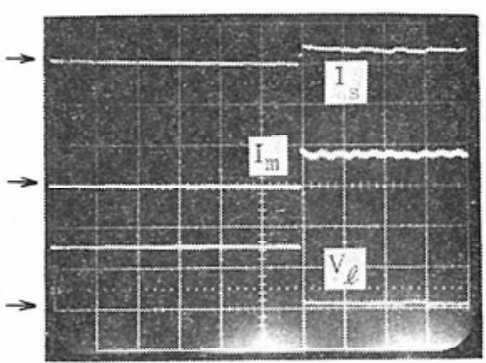

(c)

$I_{s}: 10 \mathrm{~mA} /$ div.

$I_{m}: 1 \mathrm{~A} /$ div

$V_{\imath}: 100 \mathrm{~V} / \mathrm{div}$

$V_{A c}=150 \mathrm{~V}$

$V_{A B}=150 \mathrm{~V}$

Time scale: $20 \mathrm{~ms} / \mathrm{div}$.

Fig. 4 Typical current growth and voltage collapse in the main gap where $A$ operates as a cathode. $(a),(b)$ : metal halide lamp, (c) : mercury lamp

for short period as several hours and in case of A operated as an anode rather than as a cathode. The type (2) was apt to occur in the lamp burned for long period as several hundred hours and in the measurement repeated every a few minutes.

Fig. 4(a) and (b) are the typical oscillograms showing voltage collapse and current growth of the type (1) and (2). In general, the type (1) and (2) characterized by step discharge $\mathrm{e}^{5)}$ correspond to a streamer-type and a Townsend-type breakdown, respectively.

In mercury lamp the first stage discharge with small current was not observed even at low impressed voltage and a streamer-type breakdown shown in Fig. 4(c) always occurred independently of such conditions as impressed voltage, overvoltage and burning period of the lamp.

\section{(b) In case of the main electrode $\mathbf{A}$ as an anode}

Fig. 5 shows a typical current growth when the main electrode $\mathrm{A}$ operates as an anode. The first stage discharge with small current of about $100 \mu \mathrm{A}$ appeared also in this case and it transited to the second stage discharge with increasing impressed voltage.

In this case, the type (1) shown in Fig. 6(a) was always observed in the lamp burned for short period and type (1) or (2) shown in Fig. 6(b) for long period. The type (1) shown in Fig. 6(c) was always observed independently of the conditions in mercury lamps.

All these oscillograms may suggest that the appearance of the first stage discharge and the step discharge are closely related to the lamp type, in other words, the gas composition and/or electron emissivity of the cathode.

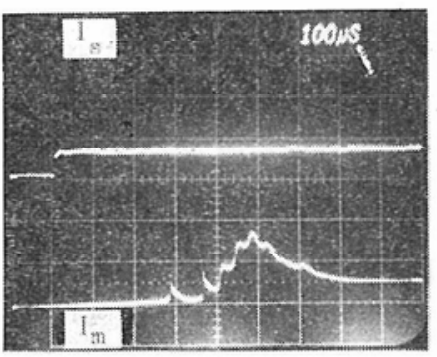

(a)

$V_{s c}=300 \mathrm{~V}$

$V_{A B}=400 \mathrm{~V}$ (overvoltage: $\sim 2 \%$ )

$I_{s}$ : probe current $(3 \mathrm{~mA} /$ div. $)$

$I_{m}$ : main current $(50 \mu \mathrm{A} /$ div. $)$

Time scale: $100 \mu \mathrm{s} /$ div.

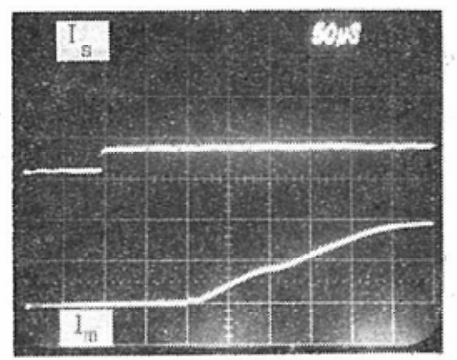

(b)

$V_{A c}=300 \mathrm{~V}$

$V_{\text {, } B}=480 \mathrm{~V}(\sim 20 \%)$

$I_{s}: 3 \mathrm{~mA} /$ div.

$I_{m}: 10 \mathrm{~mA} /$ div .

Time scale: $50 \mu \mathrm{s} /$ div.

Fig. 5 Typical current growth in the main gap where $A$ operates as an anode. 


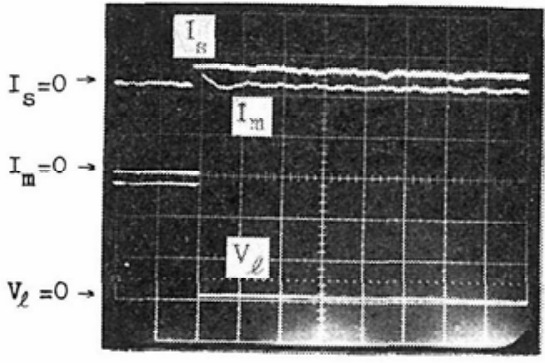

(a)

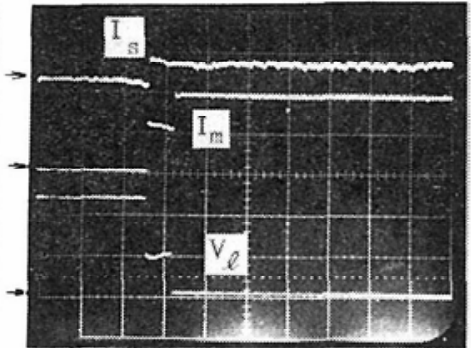

(b)

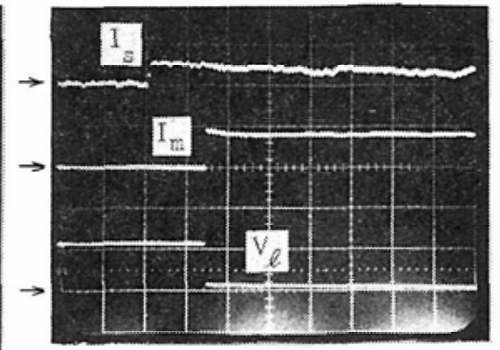

(c)

$$
\begin{aligned}
& I_{s}: 10 \mathrm{~mA} / \text { div. } \\
& I_{m}: 2 \mathrm{~A} / \text { div. } \\
& V_{t}: 200 \mathrm{~V} / \text { div. } \\
& V_{A C}=400 \mathrm{~V} \\
& V_{A B}=540 \mathrm{~V} \\
& \text { Time scale: } 10 \mathrm{~ms} / \text { div. }
\end{aligned}
$$

$$
\begin{aligned}
& I_{s}: 10 \mathrm{~mA} / \text { div. } \\
& I_{m}: 2 \mathrm{~A} / \text { div. } \\
& V_{t}: 200 \mathrm{~V} / \text { div. } \\
& V_{A C}=450 \mathrm{~V} \\
& V_{A b}=480 \mathrm{~V} \\
& \text { Time scale: } 10 \mathrm{~ms} / \text { div. }
\end{aligned}
$$

Fig. 6 Typical current growth and voltage collapse in the main gap where A operates as an anode.

\section{$(a),(b)$ : metal halide lamp, (c): mercury lamp}

\subsection{Measurements of formative time lag}

\subsubsection{Formative time lag in the starting gap}

Sparking time lag is given by

$$
\tau=\tau_{s}+\tau_{f}
$$

where $\tau s$ is the statistical time lag and $\tau$, the formative time lag. If $n$ is the total numbers of trials and $n_{\tau}$ is the number of trials where the sparking

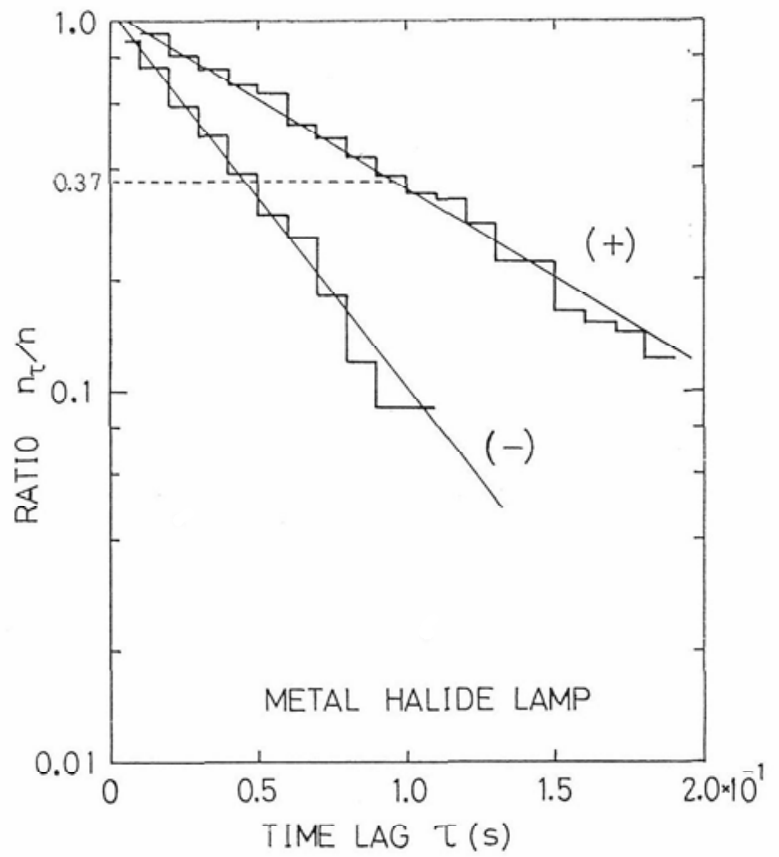

Fig. 7 Example of Laue plots in the starting gap.

$(+)$ : polarity of the probe is positive. $(-)$ : polarity of the probe is negative. Overvoltage: $5 \sim 10 \%, V_{A C}=400 \mathrm{~V}$, $R_{s}=100 \mathrm{~K} \Omega$ time lag is larger than $\tau$, the ratio $n \tau / n$ is given by

$$
\frac{n_{\tau}}{n}=\exp \left(-\frac{\tau-\tau_{f}}{\tau_{s}}\right) \text {. }
$$

$\tau_{s}$ an $\tau$ s can be determined by $\ln (n \tau / n)$ vs. $\tau$ curve (Laue plots) whieh is approximated by a straight line and hence $\tau$ becomes $\tau_{f}$ and $\tau_{f}+\tau_{s}$ assuming $n \tau / n=1$ and $n \tau / n=1 / e(=0.37)$, respectively.

Fig. 7 shows an example of Laue plots in the starting gap of the metal halide lamp. The results of $\tau_{s}$ and $\tau_{s}$ are shown in Table 1. $\tau_{s}$ generally

Table 1 Sparking time lags in the starting gap

\begin{tabular}{c|c|c|c|c}
\hline \multicolumn{1}{c|}{ Lamps } & $\begin{array}{c}\text { Polarity of } \\
\text { the probe }\end{array}$ & $\tau_{s}(s)$ & $\tau_{f}(s)$ & $\begin{array}{c}\text { Overvoltage } \\
(\%)\end{array}$ \\
\hline \multirow{2}{*}{$\begin{array}{l}\text { Metal halide } \\
\text { lamp }\end{array}$} & positive & $9 \times 10^{-2}$ & $6 \times 10^{-3}$ & \multirow{2}{*}{$5 \sim 10$} \\
\cline { 2 - 4 } & negative & $4 \times 10^{-2}$ & $3 \times 10^{-3}$ & \\
\hline \multirow{2}{*}{ Mercury lamp } & positive & $1 \times 10^{-3}$ & $7 \times 10^{-4}$ & \multirow{2}{*}{10} \\
\cline { 2 - 4 } & negative & $2 \times 10^{-4}$ & $2 \times 10^{-4}$ & \\
\hline
\end{tabular}

decreased with increasing overvoltage and was always greater than the drift time of positive ions as will be described later.

\subsubsection{Formative time lag in the main gap}

For measuring the formative time lag, we used high series resistance of $R_{m}(=10 \mathrm{~K} \Omega)$ so that the stable discharge after breakdown did not disturb the surface conditions of the cathode.

The formative time lags $\left(\tau_{f 1}\right.$ and $\left.\tau_{f 2}\right)$ of the first and the second stage discharge showed the dependence on such conditions as arc tubes, energy of the auxiliary discharge, impressed voltage and its polarity within the limits of this experiment. Fig. 8 and Fig. 9 show the relation between the impressed voltage $V_{A B}$ and time lags $\tau_{/ 1}$ and $\tau_{f 2}$ in case of A operated as a cathode. Fig. 10 and Fig. 11 are the same relation in case of A operated as an anode. In general, $\tau_{f 1}$ and $\tau_{f 2}$ showed a tendency to decrease with increasing $V_{A B}$ in both cases. In case 


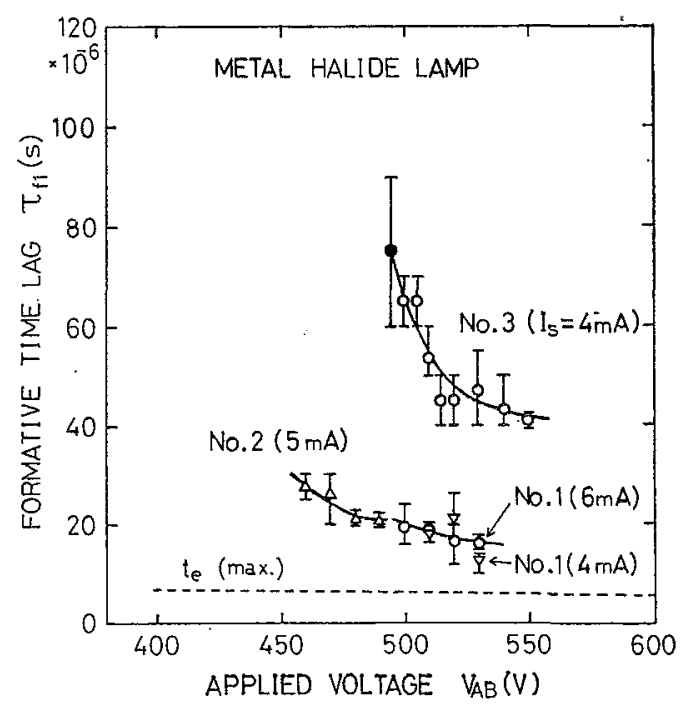

Fig. 8 Formative time lag of the first stage discharge as a function of the impressed voltage to the main gap where $A$ operates as a cathode.

- : minimum breakdown voltage.

Broken line: maximum drift time of electrons in argon.

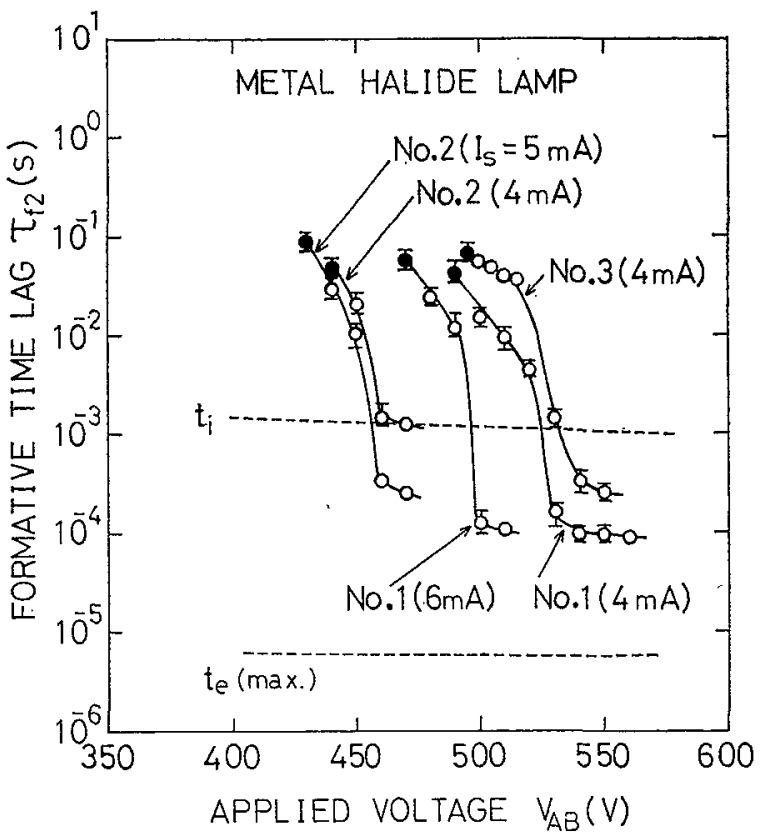

Fig. 9 Formative time lag of the second stage discharge as a function of the impressed voltage to the main gap where $A$ operates as a cathode.

- : minimum breakdown voltage.

Broken line: drift times of electrons and $\mathrm{Hg}^{+}$ ions.

of $A$ operated as a cathode, $\tau_{f 2}$ remarkably depended on $V_{A B}$ and such long time lag in order of $10 \mathrm{~ms}$ was observed at. low overvoltage. Fig. 12 shows the formative time lag $\tau_{f}$ as a function of the applied voltage in the mercury lamp.

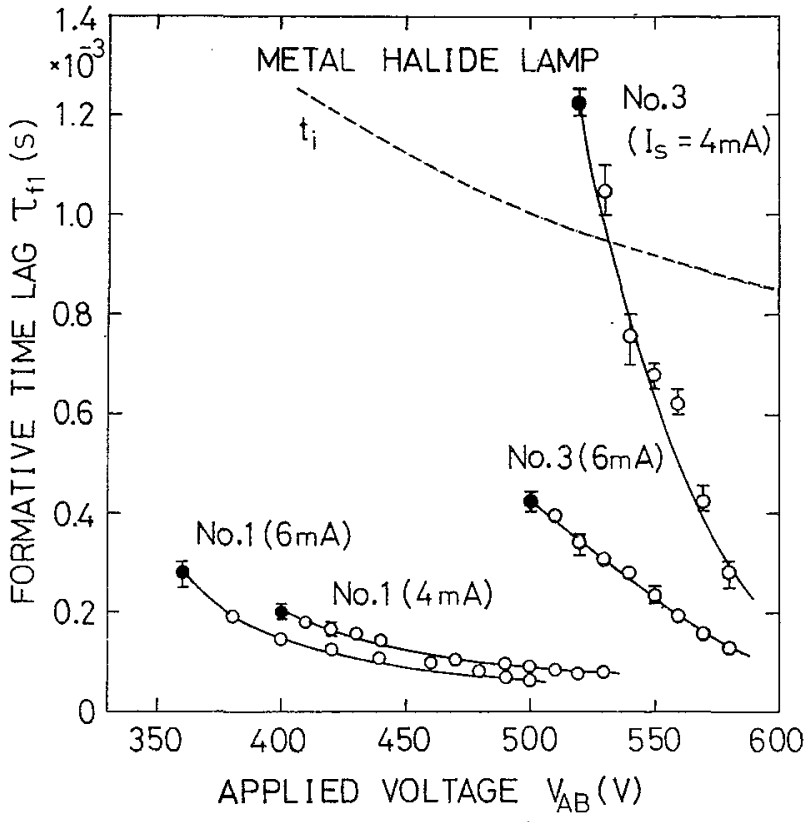

Fig. 10 Formative time lag of the first stage discharge as a function of the impressed voltage to the main gap where $A$ operates as an anode.

- : minimum breakdown voltage.

Broken line: drift time of $\mathrm{Hg}^{+}$ions in argon.

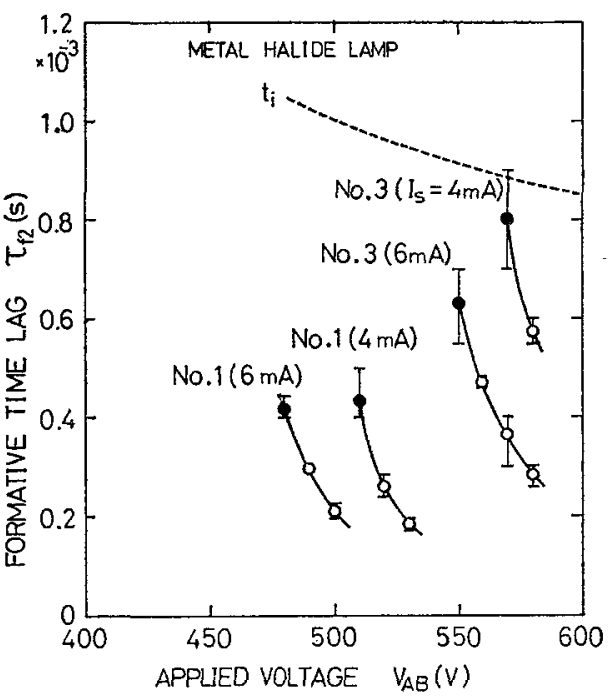

Fig. 11 Formative time lag of the second stage discharge as a function of the impressed voltage to the main gap where $A$ operates as an anode.

- : minimum breakdown voltage.

Broken line: drift time of $\mathrm{Hg}^{+}$ions in argon.

\subsection{Calculation of electronic and ionic drift times}

\subsubsection{Drift time of electrons in the main gap}

In the calculation of the drift times, we make the assumption that the density of impurity gases is negligible as compared with 35 Torr argon. At 


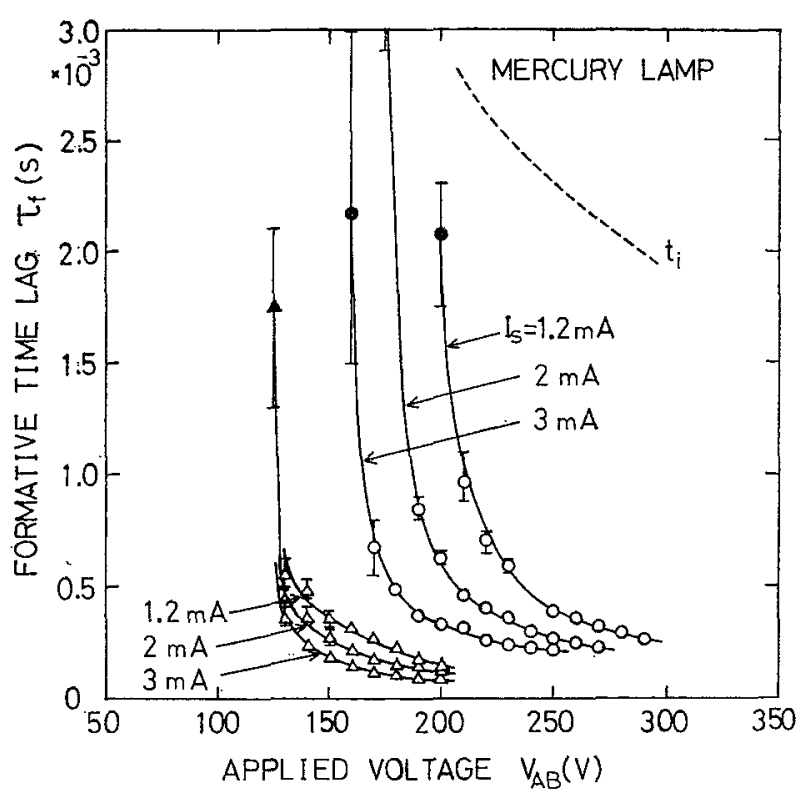

Fig. 12 Formative time lag as a function of the impressed voltage to the main gap.

$O: A$ operates as an anode.

$\triangle: A$ operates as a cathode.

Broken line: dirft time of $\mathrm{Hg}^{+}$ions in argon.

room temperature the vapor pressure of the metal halide additives and mercury are negligible as compared with argon, therefore, it is considered that electrons drift in pure argon.

In general, the drift time of electrons or positive ions is given by

$$
t_{e(i)}=\frac{d}{v_{e(i)}}
$$

where $d$ is the drift distance, $v_{e}$ and $v_{i}$ are electronic and ionic drift velocities respectively. The drift velocity is given by

$$
v_{e(i)}=\mu_{e(i)} E
$$

where $E$ is the electric field, $\mu_{\varepsilon}$ and $\mu_{i}$ electronic and ionic mobilities respectively ${ }^{\circ}$.

In the uniform field, when the electric field is applied along the direction of the axis, an electron which starts from the cathode is accelerated by the electric field and reaches a velocity equilibrium with the field after a certain time because of collisions with argon atoms ${ }^{7}$. The random velocity of the electrons has reached about 94 per cent of its final value at time $t=\tau_{e} . \tau_{e}$ is a time constant in this process and is given by

$$
\tau_{e}=\left(\frac{1}{2}\right)^{\frac{1}{4}}\left(\frac{m_{e}}{e}\right)^{\frac{1}{2}} f^{-\frac{3}{4}}\left(\frac{\lambda_{e}}{E}\right)^{\frac{1}{2}}
$$

where $f=2 m_{\theta} M_{A r} /\left(m_{\theta}+M_{A r}\right)^{2}, \lambda_{\theta}$ is the mean free path of an electron in argon, $m_{1}$ and $M_{A r}$ are the masses of an electron and an argon atom respectively $^{8}$. The drift velocity of the electrons also reaches about 94 per cent of the final velocity at $\tau_{e}$.

We obtain $\tau_{\theta}=1.57 \times 10^{-6} s$ from Eq. (5) using $E_{0}=100 \mathrm{~V} / \mathrm{cm}, f=2.72 \times 10^{-5}, e=4.8 \times 10^{-10} \mathrm{esu}, m_{e}=$ $9.1 \times 10^{-28} \mathrm{~g}$ and $\lambda_{e}=8.6 \times 10^{-2} \mathrm{~cm}^{9)}$ (at $E_{0} / P=100 / 35=$ $2.86 \mathrm{~V} / \mathrm{cm}$. Torr).

The experimental value for the electron drift velocity at $E / P=2.86$ has been reported as $v_{0} \fallingdotseq$ $1 \times 10^{6} \mathrm{~cm} / \mathrm{s}^{8)}$. The average drift velocity of the electrons is $v_{e} / 2 \doteqdot 5 \times 10^{5} \mathrm{~cm} / \mathrm{s}$ at $0 \leqq t \leqq \tau_{e}$ at the lowest estimate, so that the drift distance is about $0.8 \mathrm{~cm}$. Therefore, the drift time of the electrons across the gap is $t_{e}=0.8 / 5 \times 10^{5}+4.2 / 1 \times 10^{6}=5.77 \times$ $10^{-6} \mathrm{~s}$.

In the gap of the metal halide lamp, we must find the strength of the electric field in the main gap to obtain $t_{\theta}$ and $t_{i}$. The electric field in the main gap can be approximated with that between parallel plate electrodes with a spheroidal projection on the inner side of one of them (Fig. 13). Let $E_{0}\left(=V_{A B} / d\right)$ be the average strength of the electric field, then the electric field $E$ as a function of the distance $z$ along the axis can be expressed as follows ${ }^{10)}$ :

$$
E=E_{0}\left(1-\frac{\ln \sqrt{\frac{z+a+c}{z+a-c}-\frac{c(z+a)}{(z+a)^{2}-c^{2}}}}{\ln \sqrt{\frac{a+c}{a-c}}-\frac{c}{a}}\right),
$$

and then the derivative of $E$ with respect to $z$ is written by

$$
\frac{d E}{d z}=-E_{0}\left\{\frac{2 c^{3}}{\left(\ln \sqrt{\left.\frac{a+c}{a-c}-\frac{c}{a}\right)\left[(z+a)^{2}-c^{2}\right]}\right.}\right\} \cdots \cdots(7)
$$

where $2 a$ is the major axis of the spheroid, $2 b$ the minor axis, and $C$ is equal to $\sqrt{a^{2}-b^{2}}$.

As an example, the field strength calculated from Eq. (6) using $a=0.8 \mathrm{~cm}, b=0.12 \mathrm{~cm}, d=5 \mathrm{~cm}$ and $V_{A B}=500 \mathrm{~V}$ is illustrated in Fig. 13.

Since the electric field at the region near the pointed end of the electrode is much higher than the average field in the main gap as shown in Fig. 13, the drift time in the main gap is smaller than the value of $t_{\theta}=5.77 \times 10^{-6} \mathrm{~s}$ derived in the uniform field gap. This means that $t_{0}$ corresponds to the maximum drift time in the main gap.

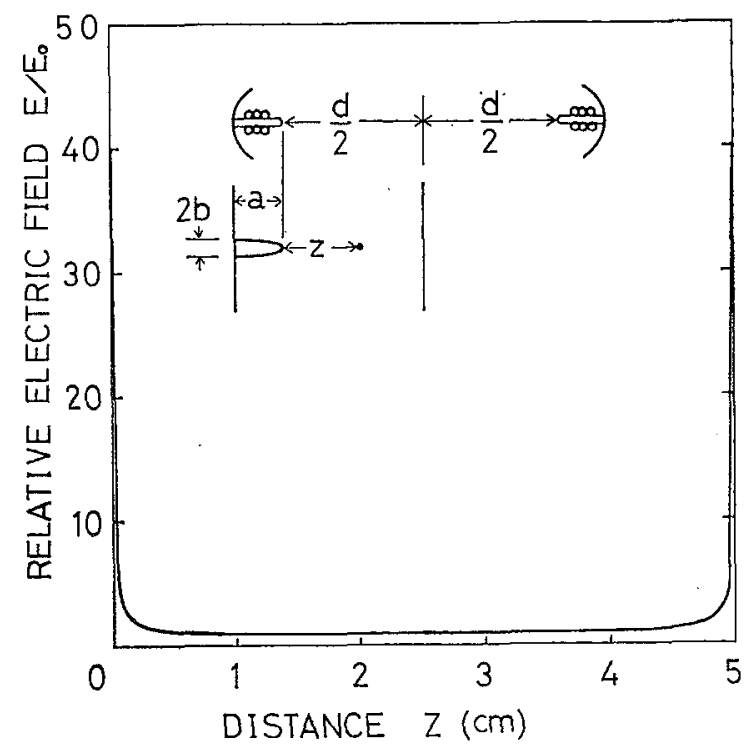

Fig. 13 Strength of the electric field in the main gap calculated approximately using parallel plate electrodes with a spheroidal projection. $E_{0}=V_{A B} / d, V_{A B}=500 \mathrm{~V}, d=5 \mathrm{~cm}$, $a=0.8 \mathrm{~cm}, b=0.12 \mathrm{~cm}$. 
Thus, we obtain the following relations comparing $\tau / 1$ and $\tau_{f 2}$ with $t_{\theta}$,

$$
\tau_{f 1}>t_{e}, \quad \tau_{f 2}>t_{e} .
$$

We can also calculate the drift time of the electrons in mercury lamps in the same way, and $t_{s} \doteqdot$ $1.4 \times 10^{-5} \mathrm{~s}$ obtained using $v_{e} \fallingdotseq 5 \times 10^{5} \mathrm{~cm} / \mathrm{s}^{3)}$ (at $E / P$ $=1.43 \mathrm{~V} / \mathrm{cm}$. Torr), $V_{A B}=200 \mathrm{~V}, P_{A}=20$ Torr and $d=$

$7 \mathrm{~cm}$. In this case, we obtain the following relation $\tau_{f}>t_{e}$.

\subsubsection{Drift time of $\mathbf{H g}^{+}$ions in the starting and main gaps \\ (a) Drift time in the starting gap}

The strength of the electric field in the starting gap can be approximated with that between parallel rod electrodes and is given by

$$
E=\frac{V_{A C}}{\ln \frac{\left(d-r_{1}\right)\left(d-r_{2}\right)}{r_{1} \gamma_{2}}}\left(\frac{1}{x}+\frac{1}{d-x}\right) \cdots \cdots \cdots \cdots(10)
$$

where $x$ is the distance from the axis of the main electrode, $V_{A C}$ is the impressed voltage, $r_{1}$ and $r_{2}$ are radii of the main and probe electrodes, and $d$ is the distance between the electrodes, respectively. The field strength calculated from Eq. (10) using $V_{A C}=400 \mathrm{~V}, r_{1}=0.12 \mathrm{~cm}, r_{2}=0.025 \mathrm{~cm}$ and $d=0.4 \mathrm{~cm}$ is illustrated in Fig. 14.

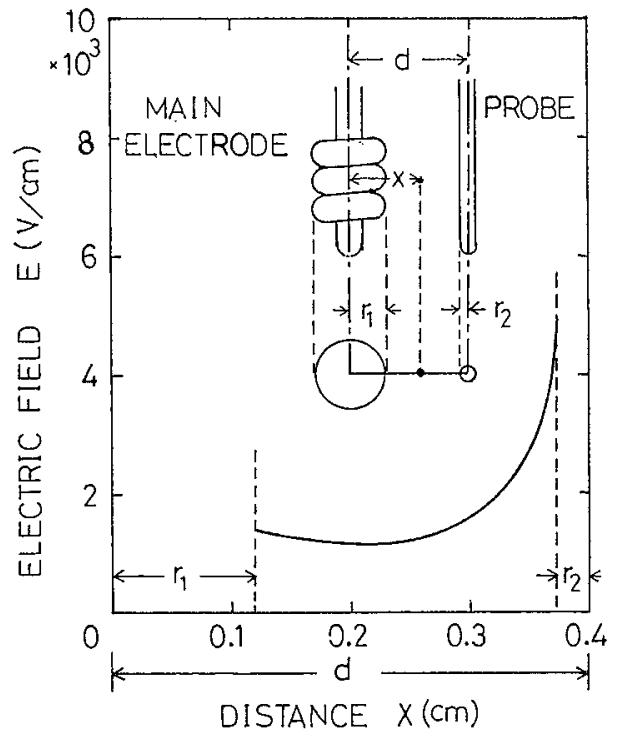

Fig. 14 Strength of the electric field in the starting gap calculated approximately using parallel rod electrodes. $V_{A C}=400 \mathrm{~V}, d=0.4 \mathrm{~cm}, r_{1}=0.12 \mathrm{~cm}$, $r_{2}=0.025 \mathrm{~cm}$.

The drift time calculated using the value $E=$ $1.13 \times 10^{3} \mathrm{~V} / \mathrm{cm}$ of the minimum field shown in Fig. 14 corresponds to the maximum drift time. The maximum drift time $t_{i}$ of $\mathrm{Hg}^{+}$ions in 35 Torr argon at $300 \mathrm{~K}$ is calculated to be $5.16 \times 10^{-6} \mathrm{~s}$ from Eqs. (3) and (4) using $E=1.13 \times 10^{3} \mathrm{~V} / \mathrm{cm}$ and $\mu_{i}=43.9 \mathrm{~cm}^{2} / V$.s which was calculated from the zero field mobility $\mu_{0}=1.84 \mathrm{~cm}^{2} / \mathrm{V} \cdot \mathrm{s}^{11)}$ of $\mathrm{Hg}^{+}$ions in 760 Torr argon at $273 \mathrm{~K}$. Thus, we obtain the following relation,

$$
\tau_{f} \gg t_{i}
$$

In the mercury lamp we can determine $t_{i}=2.03 \times$ $10^{-6} \mathrm{~s}$ in the same way using $V_{A C}=165 \mathrm{~V}, r_{1}=0.15 \mathrm{~cm}$, $r_{2}=0.02 \mathrm{~cm}$ and $d=0.3 \mathrm{~cm}$. Here we also obtain the same relation,

$$
\tau_{f} \gg t_{i}
$$

\section{(b) Drift time in the main gap}

The mean free path $\lambda_{g 0}$ of a $\mathrm{Hg}$ atom in argon $(273 \mathrm{~K}, 1$ Torr) is written by

$$
\lambda_{g o}=\frac{1}{\sqrt{2 \pi N_{g}\left(r_{H g}+r_{A r}\right)^{2}}}=5.32 \times 10^{-3} \mathrm{~cm} \cdots(13)
$$

where $N_{g}\left(=3.536 \times 10^{16} \mathrm{~cm}^{-3}\right)$ is the atomic density of argon at 1 Torr and $273 \mathrm{~K}, r_{H g}(=1.55 \AA)$ and $\gamma_{A \cdot r}(=1.91 \AA)$ are the atomic radii ${ }^{12}$ of mercury and argon respectively. $\lambda_{\rho 0}$ is converted to $\lambda_{g}=1.67 \times$ $10^{-4} \mathrm{~cm}$ at 35 Torr of argon and $300 \mathrm{~K}$

As the time constant $\tau_{i}\left(\approx 10^{-8} \mathrm{~s}\right.$ of $\mathrm{Hg}^{+}$ions in argon calculated from Eq. (5) is much smaller than $\tau_{0}, \tau_{i}$ can be neglected as compared with the drift time of $\mathrm{Hg}^{+}$ions across the gap.

Using the mean free path of a $\mathrm{Hg}^{+}$ion as $\lambda_{i}$ $\left(=\sqrt{2} \lambda_{l}=2.36 \times 10^{-4} \mathrm{~cm}\right)$, the ratio $(d E / d x) \lambda_{i} / E$ at the higher field strength region near the top of the main electrode is calculated to be $-1.47 \times 10^{-2}$ (1.47\%) from Eqs. (6) and (7). Therefore, $(d E / d x) \lambda_{i}$ is found to be negligible as compared with $E$. In this case, as the drift velocity of $\mathrm{Hg}^{+}$ ions is considered to be in equilibrium with the electric field, the drift time of $\mathrm{Hg}^{+}$ions can be calculated from the equation

$$
\begin{aligned}
t_{i} & =\int_{0}^{a} \frac{1}{v_{i}} d x \\
& =\frac{2}{\mu_{i}} \int_{0}^{a / 2} \frac{1}{E} d x .
\end{aligned}
$$

Thus, we obtain $t_{i}=1.02 \times 10^{-3} \mathrm{~s}$ using $V_{A B}=500 \mathrm{~V}$, $\mu_{i}=43.9 \mathrm{~cm}^{2} / \mathrm{V} . \mathrm{s}, d=5 \mathrm{~cm}, a=0.8 \mathrm{~cm}$ and $b=0.12 \mathrm{~cm}$. Also for mercury lamps, we obtain $t_{i}=2.89 \times 10^{-3} \mathrm{~s}$ using $V_{A B}=200 \mathrm{~V}, \mu_{i}=76.8 \mathrm{~cm}^{2} / \mathrm{V} . \mathrm{s}$ (where argon pressure equals 20 Torr), $d=7 \mathrm{~cm}, a=1.0 \mathrm{~cm}$ and $b=0.15 \mathrm{~cm}$.

From the data described above, we lead to the following relations for the metal halide lamp:

(1) In case of the main electrode $A$ operating as a cathode,

$$
\begin{array}{lll}
t_{i} \gg \tau_{f 1}, & \tau_{f 2} \geqq t_{i} \quad \text { (at lower overvoltage) } \\
& \tau_{f 2} \leqq t_{i} \quad \text { (at higher overvoltage) } \cdots(15)
\end{array}
$$

(2) In case of $A$ operating as an anode, $t_{i} \gtrsim \tau_{f 1}, \quad t_{i} \gtrsim \tau_{f 2} . \quad \cdots \ldots \ldots \ldots \ldots \ldots \ldots \ldots \ldots \ldots \ldots(16)$

And for the mercury lamp, we obtain

$$
t_{i} \geqq \tau_{f} \text {. }
$$

\subsection{Dominant secondary process}

The $\alpha$ process alone can not produce breakdown and the secondary process must participate in the further multiplication of the electron current. This process may occur in the gas ( $\beta$ process) or at the cathode surface ( $\gamma$ process). 
The dominant secondary process in the uniform field gap can be determined by comparing the formative time lag with the drift times as follows:

(a) $\tau$ $\leqq t$

$\beta_{p}$ process (photo-ionization in gas)

(b) $t_{e}<\tau_{j}<t_{i}$ :

$\gamma_{p}$ process (secondary electron emission by photon)

(c) $t_{i}<\tau$,

$\gamma_{i}$ process (secondary electron emission by positive ions).

According to the report $t^{3)}$, as both the gaps of the metal halide lamp and the mercury lamp are considered to be the quasi-uniform field, we can roughly presume the dominant secondary process using the judging method described above. It is summarized in Table 2.

Table 2 Dominant secondary process

\begin{tabular}{|c|c|c|c|c|}
\hline \multicolumn{2}{|c|}{ Discharge gap } & \multicolumn{2}{|c|}{ Metal halide lamp } & Mercury lamp \\
\hline \multicolumn{2}{|c|}{ Starting gap } & \multicolumn{2}{|l|}{$\gamma_{i}$} & $\gamma i$ \\
\hline \multirow{4}{*}{ Main gap } & \multirow{2}{*}{$\begin{array}{l}\text { Electrode } A \text { as } \\
\text { a cathode }\end{array}$} & $\begin{array}{l}\text { The first stage } \\
\text { discharge }\end{array}$ & $r_{p}$ & \multirow{2}{*}{$r_{p}$} \\
\hline & & $\begin{array}{l}\text { The second stage } \\
\text { discharge }\end{array}$ & $\gamma_{i}, \gamma_{p}$ & \\
\hline & \multirow{2}{*}{$\begin{array}{l}\text { Electrode } A \text { as } \\
\text { an -anode }\end{array}$} & $\begin{array}{l}\text { The first stage } \\
\text { discharge }\end{array}$ & $\gamma p$ & \multirow{2}{*}{$\gamma p$} \\
\hline & & $\begin{array}{l}\text { The second stage } \\
\text { discharge }\end{array}$ & $\gamma p$ & \\
\hline
\end{tabular}

\section{Discussion}

Positive ions are formed easily at the higher field strength region near the electrodes. They also are formed even at the lower field strength region of the arc tube, because ionization index at the lower field strength region, calculated using $\alpha / E$ vs. $E / P$ characteristics $^{13)}$ for gas mixtures of metal halide lamps and Fig. 13, can not be neglected as compared with that at the higher field strength region. This means that positive ions are formed all over the gap.

By the reason of the fact described above, the drift distance for positive ions was represented by the gap length in Eq. (14). The drift time $t_{i}$, calculated from Eq. (14), corresponds to the maximum one. So, it is obvious that the dominant secondary process is $\gamma_{i}$ process, when the formative time lag is much longer than $t_{i}$. If positive ions whose drift distance is shorter than the gap length have an effect on the breakdown, then it can be considered that $\gamma_{i}$ process also in addition to $\gamma_{p}$ process participates in the breakdown, when the formative time lag is longer than $t_{e}$ and shorter than $t_{i}$.

According to the fact that $\tau s$ is much longer than $t_{i}$ in the starting gap in the metal halide and mercury lamp, it is clear that a Townsend type breakdown with $\gamma_{i}$ process as the dominant secondary process takes place as shown in Table 2 .

It seems that the breakdown mechanism in the main gap is much complicated because of the presence of the auxiliary discharge. The breakdown in the main gap is initiated by that in the starting gap. This means that an initial electron emission in the main gap is caused by the auxiliary discharge. When the main electrode $A$ operates as an anode, the initial electron is emitted from the cathode B by UV radiation generated from the discharge. When $A$ operates as a cathode, plasma due to the discharge is localized near the cathode. In this case, it is strongly possible that the initial electron may be extracted by the anode $B$ from the plasma. Therefore, we can not conclude that the dominant secondary process for the first stage discharge is $\gamma_{p}$ process as shown in Table 2 in this case.

In the mercury lamp, the initial electron was emitted easily and rapidly led to the breakdown at even lower impressed voltage $V_{A B}$. This fact suggests that the sufficiently large electron multiplication is produced because of the high emissive cathode and the Penning mixtures with the high ionization coefficient.

On the contrary, the following two types of breakdown were observed in the metal halide lamp: (1) prebreakdown $\rightarrow$ arc and (2) prebreakdown $\rightarrow$ glow $\rightarrow$ arc. The prebreakdown stage and the glow stage in the type (2) were observed in the metal halide lamp alone. These facts suggest that there are some factors suppressing electron multiplication. One of the factors is considered to be the effective ionization coefficient. It decreases due to electron attachment reaction and/or quenching reaction in Penning mixtures containing metal halide vapor and impurity gases ${ }^{13)}$.

The formative time lag $\tau_{f 2}$ was $1 \mathrm{~ms}$ at the most as shown in Fig. 11 in case of $A$ operated as an anode. In case of $A$ operated as a cathode, however, the longer $\tau_{j 2}$ as about $100 \mathrm{~ms}$ was observed as shown in Fig. 9. Such long $\tau_{f 2}$ indicates the possibility of another factor, for example formation of negative field, in addition to the factor described above. It is considered that the negative field is formed in the gap between the anode and the positive space charge accumulated near the cathode due to the auxiliary discharge.

The appearance of the glow-to-arc step discharge in the type (2) also suggests that the electron multiplication is suppressed by reduction of the effective ionization coefficient due to electron attachment in impurity gases. The type (2) clearly corresponds to a Townsend-type breakdown because of the appearance of the glow stage.

On the contrary, the type (1) in the metal halide lamp can be explained by the multiple avalanceto-streamer transition due to Townsend build-up ${ }^{14)}$ which means that the space charge effect grows by the generation mechanism. The breakdown in the mercury lamp also can be explained by the same model.

In summary, Townsend mechanism with $\gamma_{i}$ process and/or $\gamma_{p}$ process as the dominant secondary process has an important role for the breakdown in the metal halide lamp.

\section{Conclusion}

The current growth, the voltage collapse and the 
formative time lag were measured. The time lag was compared with the calculated value of electronic and ionic drift time in order to presume the dominant secondary process. The breakdown mechanism was discussed on the basis of these data.

The results are summarized as follows:

In the starting gap of both metal halide and mercury lamps, a Townsend-type breakdown occurred with $\gamma_{i}$ process as the dominant secondary process.

An initial electron emission in the main gap is caused by the auxiliary discharge in both lamps.

In the main gap of the metal halide lamp, the first stage discharge (prebreakdown stage) with small current appeared at lower impressed voltage and transited to the breakdown with increasing the impressed voltage. The following two types of breakdown were observed: (1) prebreakdown $\rightarrow$ arc and (2) prebreakdown $\rightarrow$ glow $\rightarrow$ arc. Which type will occur depends on the burning period, the impressed voltage and its polarity.

On the contrary, in the mercury lamp the first stage did not appear and the voltage collapse similar to a streamer-type breakdown was observed independently of the conditions.

The appearance of the first stage and the step discharge is considered to be due to the suppression of electron multiplication by the electron attachment and/or quenching in Penning mixtures containing metal halide vapor and impurity gases.

Townsend mechanism with $\gamma_{i}$ process and/or $\gamma_{p}$ process as the dominant secondary process has an important role for the breakdown in the metal halide lamp.

\section{Acknowledgements}

The author would like to acknowledge the continuing guidance and encouragement of Professor R. Itatani of Kyoto University. The author also would like to thank Dr. T. Noguchi of Kyoto University, Dr. M. Kambara, Mr. J. Murai and Mr. S. Itoh of NEC Sylvania Corp. for their helpful suggestions.

\section{References}

(1) J.F. Waymouth: "Electric Discharge Lamps", Cambridge, M. I. T. Press (1971)

(2) H. Washimi: J. Illum. Engng Inst. Japan, 61 (1977) 218 (In Japanese)

(3) A. Inouye, Y. Terashita and K. Ogawa: J. Light \& Vis. Env. 1-1 (1977) 24

(4) M. M. Kekez, M. R. Barrault and J. D. Craggs: J. Phys. D: Appl. Phys. 3 (1970) 1886

(5) K. R. Allen and K. Phillips: Proc. Roy. Soc. A, 278 (1963) 188

(6) L. B. Loeb: "Basic Processes of Gaseous Electronics", University of California Press (1961)

(7) S. C. Brown: "Introduction to Electrical Discharge in Gases", John Wiley \& Sons (1966)

(8) A. Von Engel: "Ionized Gases", Oxford University Press (1965)

(9) S. C. Brown: "Basic Data of Plasma Physics", M. I. T. Press (1966)

(ini) J. Hara, T. Nitta, K. Kawane and N. Yamada: Mitsubishi Denki Giho, 37 (1963) 873 (In Japanese)

(11) L. M. Chanin and M. A. Biondi: Phys. Rev. 107 (1957) 1219

(12) D. E. Gray ed.: "American Institute of Physics Handbook", McGraw-Hill (1972)

(13) H. Washimi: J. Illum. Engng Inst. Japan, 59 (1975) 590 (In Japanese)

(14) Y. Miyoshi: Phys. Soc. Japan, 30-8 (1975) 591 (In Japanese)

(1977. 7. 11, 1977. 10.11) 